\title{
EXCITATORY AND INHIBITORY ACTIVITY IN THE DORSAL MUSCULATURE OF THE NEMATODE ASCARIS EVOKED BY SINGLE DORSAL EXCITATORY MOTONERONS ${ }^{1}$
}

\author{
J. P. WALROND ${ }^{2}$ AND A. O. W. STRETTON ${ }^{3}$ \\ Department of Zoology, University of Wisconsin, Madison, Wisconsin 53706
}

Received May 28, 1981; Revised July 23, 1984; Accepted July 25, 1984

\begin{abstract}
A physiological preparation, in which identified motoneurons of the nematode Ascaris lumbricoides can be individually stimulated, was used to map the response evoked by single dorsal excitatory (DE) moloneurons in muscle cells innervated along the length of the dorsal nerve cord. As previously reported (Walrond, J. P., I. S. Kass, A. O. W. Stretton, and J. E. Donmoyer (1985) J. Neurosci. 5: 1-8), stimulation of a DE cell produces excitatory responses in muscle cells which it directly innervates. Excitatory activity propagates along the most strongly activated region of muscle at a velocity of $\sim 28 \mathrm{~cm} / \mathrm{sec}$, then relaxes into a slower velocity of $\sim 12 \mathrm{~cm} /$ sec. When either the DE1 or DE3 neurons were stimulated, excitatory responses were also observed in muscle cells not directly innervated by the neuron. These signals propagate in the opposite direction from the fastpropagating activity at a velocity of $\sim 13 \mathrm{~cm} / \mathrm{sec}$. Injection of hyperpolarizing current into muscle cells blocks this slower propagation but fails to block the faster conduction. We conclude that the fast-conducting responses result from signals propagating in the motor axon, whereas the slow responses are conducted through gap junctions which connect Ascaris muscle cells. Stimulating a single DE motoneuron also evokes hyperpolarizing muscle responses in regions adjacent to the zones of fast and slow excitation. Anatomically defined synapses from the DE motoneurons onto the dorsal inhibitor (DI) can account for this inhibition. DE-DI synaptic interactions combined with the reciprocal inhibitory circuit discussed in the preceding paper (Walrond, J. P. and A. O. W. Stretton (1985) J. Neurosci. 5: 9-15) provide the basis for a model which describes how a propagating wave of muscle contraction could be produced during nematode locomotion.
\end{abstract}

\begin{abstract}
The goal of our studies on the motor nervous system of Ascaris lumbricoides is to explain how this nematode uses its nervous system to coordinate movement. In its natural environment, the small intestine of the pig, Ascaris braces itself against the walls of the intestine by throwing its body into a waveform consisting of dorsoventral bends. When this waveform is propagated along the body, locomotion occurs. Behavioral experiments have demonstrated that initiation and propagation of anteriorly propagating waves continue after the head and the tail are excised (see Crofton, 1971). This result indicates that the head and tail ganglia are not required for this form of coordinated locomotion and implies that the nervous system that remains, namely, the motor nervous system that is located in the body of the animal, is sufficient to produce the behavior.
\end{abstract}

\footnotetext{
${ }^{1}$ We thank A. Chambers, A. Aaroen, C. Hughes, and D. Chandler for their help in preparing the manuscript. We also thank Dr. C. D. Johnson for the benefit of many stimulating discussions, and both Dr. Johnson and Dr. C. Kung for critical reading of the manuscript. This work was supported by United States Public Health Service Grants NS 10509 and AI 15429, National Science Foundation Grant BNS 7609641, a Sloan Fellowship to A. O. W. S., and the Research Fund of the Graduate School, University of Wisconsin, Madison.

${ }^{2}$ Present address: Laboratory of Neurobiology, National Institute of Neurological and Communicative Disorders and Stroke, Marine Biological Laboratory, Woods Hole, MA 02543.

${ }^{3}$ To whom correspondence should be addressed.
}

The muscle cells responsible for propagating the waveform are divided into dorsal and ventral fields. All muscle cells within a field have a noncontractile "arm" (Rosenbluth, 1965) which extends to the appropriate (ventral or dorsal) nerve cord, where they interdigitate with arms of other muscle cells, form gap junctions (Rosenbluth, 1965), and receive chemical synapses from the motoneurons. There are seven types of motoneurons (Stretton et al., 1978); three synapse onto ventral muscle cells and four synapse onto dorsal muscle cells (see Fig. 9, in Walrond et al., 1985). The dorsal cord consists almost exclusively of branches of motoneurons that are connected to their cell bodies by semicircumferential fibers called commissures (see Fig. 1 in Walrond et al., 1985). Besides the cell bodies and processes of motoneurons, the ventral cord also contains 6 large interneurons that make synapses onto the dendrites of excitatory motoneurons, and approximately 12 small interneurons that synapse onto motoneurons as well as onto large and small interneurons (J. E. Donmoyer, P. A. Desnoyers, and A. O. W. Stretton, unpublished observation).

In previous electrophysiological experiments (Walrond et al., 1985), we have determined that three types of motoneurons are dorsal excitors (DE) and two types are inhibitors, one to dorsal muscles (DI) and the other to ventral muscles (VI). We have also analyzed the synaptic connections from the DE motoneurons to the VI motoneurons (Walrond and Stretton, 1985). These connections can be used to produce a type of reciprocal 
inhibition which results in body postures like those involved in nematode locomotory movements.

In this paper we examine further the effects of ventral stimulation of single DE motoneurons by mapping the response evoked in dorsal muscle cells at different positions along the worm. The results obtained allow us to calculate the conduction velocity along individual neuronal processes and to determine the velocity with which excitatory signals propagate through the network of muscle cell gap junctions. They also show that stimulating a single DE motoneuron evokes inhibitory muscle responses in regions adjacent to the zones of excitation. We believe that the inhibitory responses are mediated by synapses from the DE motoneuron onto the DI motoneurons; numerous synapses of this type have been found by electron microscopy (P. A. Desnoyers, J. E. Donmoyer, and A. O. W. Stretton, unpublished observation). By combining these results with interactions between the DE and VI neurons described in the preceding paper (Walrond and Stretton, 1985), we have constructed a model for the generation of waveforms along the body of nematodes.

\section{Materials and Methods}

The methods for collecting and storing animals and the electrophysiological recording techniques used are similar to those previously described (Walrond et al., 1985).

Each experiment employed a preparation in which the dorsal and ventral muscle halves remained connected by a single intact commissure of a DE motoneuron (see Fig. 2 of Walrond et al., 1985). Commissures were visualized, identified, and marked as previously described (Walrond et al., 1985). The intact neuron was stimulated ventrally, and responses were recorded intracellularly in dorsal muscle cells. Small responses were revealed by averaging a number of responses utilizing a Tracor Northern digital signal averager.

In order to construct maps of response latency versus muscle cell position along the dorsal nerve cord, oscilloscope traces of the evoked responses were photographed, and the latencies between stimulus and time to first discernible response were measured directly from the photographs. To minimize variation in latency resulting from signal conduction within the muscle cell, all recordings were taken from muscle cell bodies lying immediately adjacent to the nerve cord. The distance between the impaled muscle cell and the point where the commissure entered the dorsal nerve cord was determined by measuring with a calibrated eyepiece. Plotting distance against the corresponding latency yielded a plot whose slope is the conduction velocity.

Ascaris motoneurons are arranged in five repeating units or segments. The segment to which an individual neuron belongs is indicated by the subscript Roman numerals I to V. Each segment contains 11 motoneurons including one DE2, DE3, and DI neuron each, and two DE1, VI, V-1, and V-2 neurons, each. They are distinguished by superscripts (e.g., $\mathrm{VI}^{1}{ }_{\mathrm{III}}$ and $\mathrm{VI}^{2}{ }_{\mathrm{III}}$ are the anterior and posterior $\mathrm{VI}$ neurons in the third segment).

\section{Results}

The DE motoneurons extend processes in the dorsal nerve cord either anteriorly (DE1 and DE3) or posteriorly (DE2) from the commissure (see Figs. 1 and 9 of Walrond et al., 1985). These processes make excitatory synapses to the dorsal musculature (Walrond et al., 1985). When a single DE1 or DE3 motoneuron was activated ventrally, excitatory responses were evoked in the muscles directly innervated by the axons of these neurons. Excitatory responses were also found in the noninnervated region posterior to the commissure. In both innervated and noninnervated regions, the latency increased with increasing distance from the commissure; however, activity propagated more slowly in the noninnervated region. For example, in the case of the DE1 neuron shown in Figure 1, the posteriorly propagating signals had a linear conduction velocity of about $12 \mathrm{~cm} / \mathrm{sec}$. The anteriorly propagating signals appear to fit two straight lines. In the region proximal to the commissure, signals propagated at about $25 \mathrm{~cm} / \mathrm{sec}$; more distally they slowed to

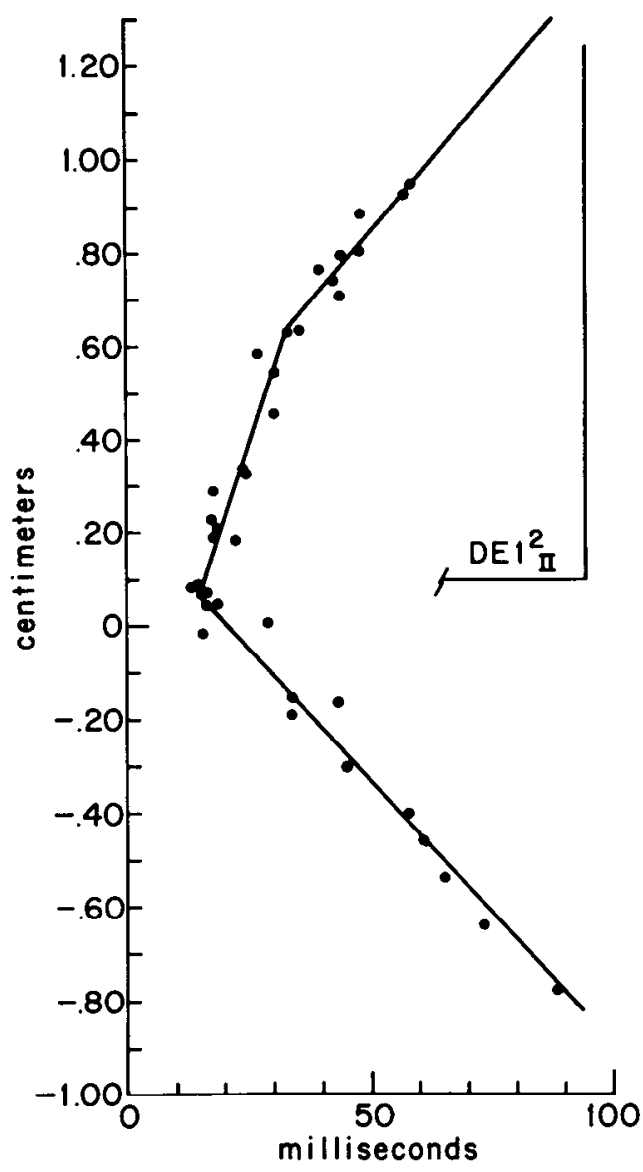

Figure 1. Map of responses produced by the activation of a DE1 neuron. Intracellular recordings were taken from muscle cells both anterior and posterior to the commissure of the $\mathrm{DE} 1^{2}{ }_{11}$ neuron. Ventrally activating the DE1 neuron evoked depolarizing potentials both anterior and posterior to the commissure. Each point represents a recording taken from a different muscle cell. The origin was placed approximately at the position of the commissure; positive distances on the ordinate are anterior; negative distances are posterior. The latency between the stimulus and the inflexion beginning the response is plotted on the abscissa. The latency near zero distance is presumably due to the synaptic delay and the conduction time along the commissure. The diagram to the right shows the position of the commissure and the anteriorly projecting dorsal process of the DE1 neuron. Anterior to the commissure there is a region of fast conduction $(25 \mathrm{~cm} / \mathrm{sec})$ followed by a region of slow conduction $(14 \mathrm{~cm} / \mathrm{sec})$. Posterior to the commissure, a region of slow conduction $(12 \mathrm{~cm} / \mathrm{sec})$ is found.

about $14 \mathrm{~cm} / \mathrm{sec}$. Four additional experiments on DE1 neurons and one of two experiments on DE3 neurons showed similar results, as shown in Table I. In experiments on DE2 neurons, posteriorly propagating signals spread at about $28 \mathrm{~cm} / \mathrm{sec}$ from the point where the commissure enters the cord, but more distally they relaxed into a conduction velocity of about $13 \mathrm{~cm} /$ sec (Fig. 2). Only one of four experiments on DE2 neurons (Table I) showed detectable propagation of slow signals in the direction opposite to the fast propagation. In two experiments on inhibitory motoneurons, hyperpolarizing responses propagated at a rate similar to that of the fast excitatory responses (Table I).

Anatomical investigations at both the light and electron microscope level (Stretton et al., 1978) indicated that each motoneuron has a neuromuscular output zone which innervates a distinct region of muscle. For all three types of $\mathrm{DE}$ motoneurons, fast conducting signals are recorded in muscle cells directly innervated by the stimulated motoneuron. These results 
TABLE I

Conduction velocities of activities propagating through muscle upon stimulation of single motoneurons

The first column lists the type of neuron investigated in each experiment. The second column gives the conduction velocity of activity propagating along the neuron. The third column gives the conduction velocity of more slowly propagating signals, beyond the fast conduction region, that travel in the same direction as the fast propagating signals. The fourth column lists the conduction velocities recorded for activity conducted in the opposite direction through the electrically coupled muscle cells not directly innervated by the neuron. Data for the DI neurons are given at the bottom of the table.

\begin{tabular}{|c|c|c|c|}
\hline \multirow{2}{*}{$\begin{array}{l}\text { Neuron } \\
\text { Type }\end{array}$} & \multicolumn{2}{|c|}{$\begin{array}{l}\text { Signals Propagating in Muscles } \\
\text { that Overlap Neuronal Process }\end{array}$} & \multirow{2}{*}{$\begin{array}{c}\text { Signals Propagating in } \\
\text { Muscles that Do Not } \\
\text { Overlap Neuronal } \\
\text { Process } \\
\begin{array}{c}\text { Slow conduction } \\
\text { velocity }\end{array}\end{array}$} \\
\hline & $\begin{array}{l}\text { Fast conduction } \\
\text { velocity }\end{array}$ & $\begin{array}{l}\text { Slow conduction } \\
\text { velocity }\end{array}$ & \\
\hline \multicolumn{4}{|c|}{$\mathrm{cm} / \mathrm{sec}$} \\
\hline DE1 & 25 & 14 & 12 \\
\hline DE1 & 27 & 14 & 11 \\
\hline DE1 & 31 & 12 & 12 \\
\hline DE1 & 30 & 11 & 15 \\
\hline DEl & 27 & 11 & 13 \\
\hline DE2 & 28 & 13 & \\
\hline DE2 & 27 & 10 & \\
\hline DE2 & 28 & 12 & \\
\hline DE2 & 38 & 13 & 13 \\
\hline DE3 & 24 & 13 & 13 \\
\hline DE3 & 28 & & 13 \\
\hline Average $\pm \mathrm{SD}$ & $28.4 \pm 3.7$ & $12.3 \pm 1.3$ & $12.7 \pm 1.2$ \\
\hline DI & 25 & & \\
\hline $\begin{array}{l}\text { DI (anterior } \\
\text { limb) }\end{array}$ & 24 & & \\
\hline $\begin{array}{l}\text { DI (posterior } \\
\text { limb) }\end{array}$ & 28 & & \\
\hline
\end{tabular}

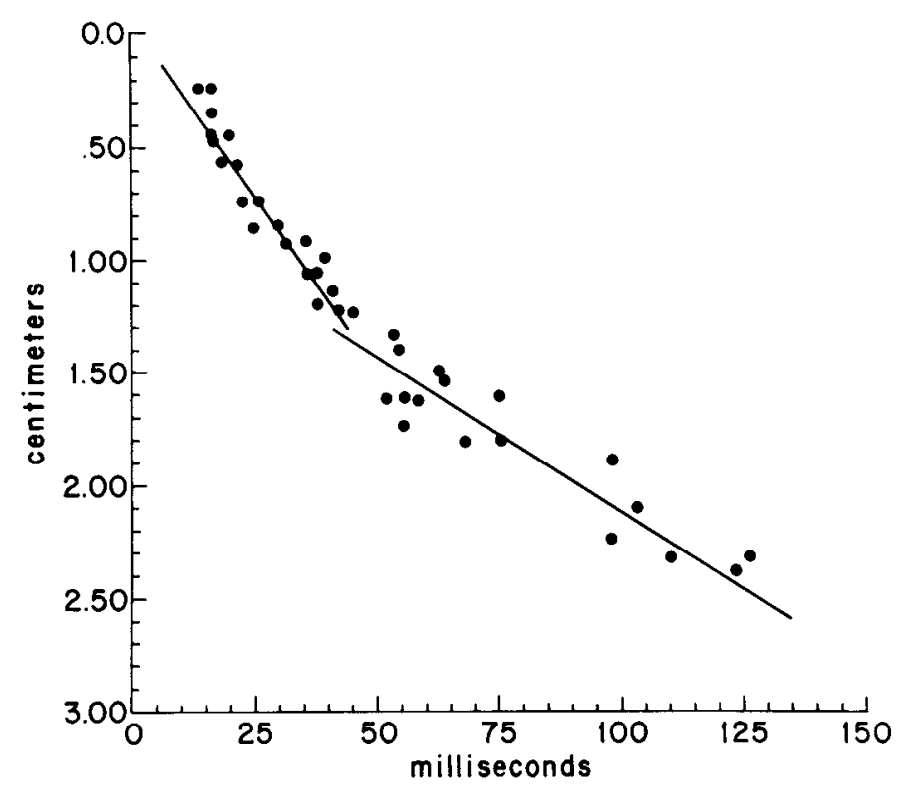

Figure 2. Map of response produced by aclivation of a DE2 neuron. Ventral activation of the $\mathrm{DE} 2 \mathrm{I}$ motoneuron evoked depolarizing potentials posterior to the commissure. Plotting the latency of these responses reveals a region of fast conduction just posterior to the commissure and a more distal region of slow conduction. imply that the propagation of activity along the intact neuron produces the region of fast conduction.

We suspected that the excitatory responses recorded in the slow conducting regions result from activity conducted through the gap junctions which interconnect the terminal arborization of muscle arms where muscle action potentials are initiated (Rosenbluth, 1965; de Bell et al., 1963; del Castillo et al., 1967). The system of muscle processes linked by gap junctions, which is classically called the "syncytium" (an incorrect term, since there is electrical but not cytoplasmic continuity between muscle cells), forms a conduction pathway along the length of the animal, which is parallel to the neurons. If the slower velocity signals are muscle action potentials carried by this pathway, then injecting hyperpolarizing current into muscle cells lying along the path of slow conduction should block the slowly propagating wave of activity by keeping a critical number of cells below threshold. On the other hand, injecting hyperpolarizing current into muscle cells along the region of fast conduction should locally hyperpolarize muscle cells without affecting activity conducted through the neuron. Neuronally conducted signals should conduct beyond the region of hyperpolarized muscle and synaptically activate more distally located muscle cells. The data presented in Figures 3 and 4 confirm these predictions. These results further indicate that the region of fast conduction results from the conduction of signals along the neuron and are consistent with the view that the region of slow conduction results from the conduction of activity from one muscle cell to the next through the system of electrically coupled muscle cells.

In order to prevent propagation through the muscle cells, these experiments required the injection of large amounts of
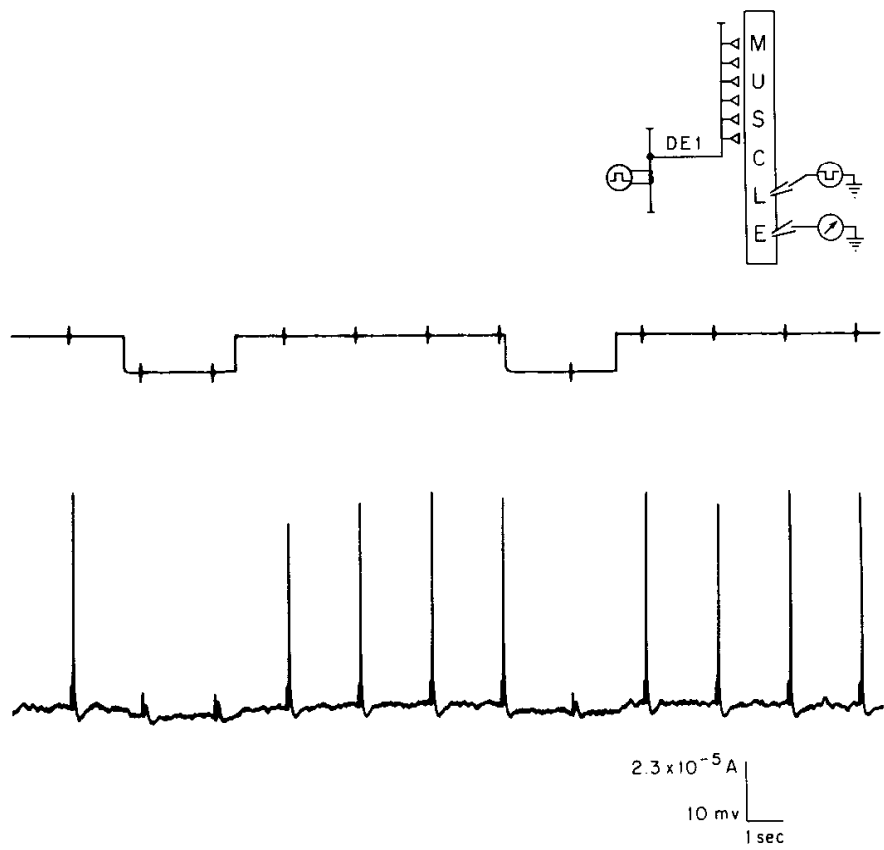

Figure 3. Injecting hyperpolarizing current blocks the spread of posteriorly conducted activity produced by the DE1 motoneuron. Posteriorly propagating responses evoked by stimulation of $\mathrm{DE}^{2}{ }_{\text {II }}$ are blocked by injecting hyperpolarizing current into muscle cells. The inset depicts the relative position of the current injecting (upside down square wave) and stimulating and recording microelectrodes. The recording site was $2.5 \mathrm{~mm}$ posterior to the current injection site. The top trace is the current monitor and the bottom trace records the muscle response. The vertical scale shows calibrations for top and bottom traces, respectively. Note that, during the hyperpolarizing pulses, although the action potentials are blocked, small depolarizing responses still occur; presumably these are due to passive current flow from unblocked muscles. 


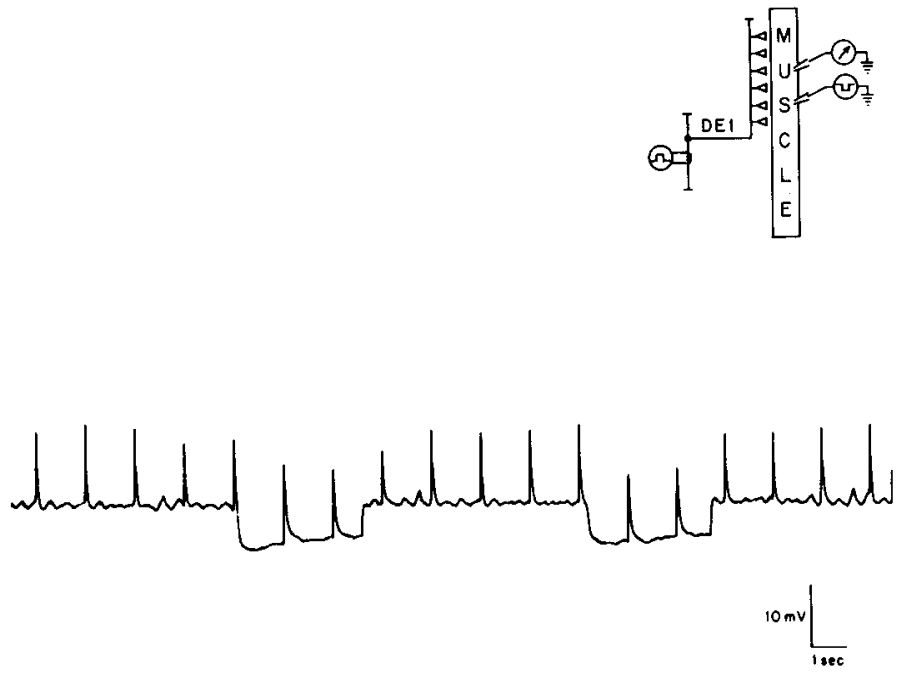

Figure 4. Injecting current into muscle cells fails to block anterior conducting activity produced by the DE1 motoneuron. In this experiment both the recording and current-injecting electrodes impaled cells in the fast conduction region. The current was injected through an electrode posterior to the recording electrode and failed to block fast conducting activity.

current (Figs. 3 and 4). The shape of the muscle cells provides an unfavorable situation for delivering current injected into the cell body to the terminal arborizations of the muscle arms. The large surface area of the bag-like cell body and the cylindrical contractile portion shunts current away from the muscle arms and their terminal arborizations (de Bell et al., 1963). Consequently, only a small proportion of the injected current reaches the muscle terminals which are the sites where muscle action potentials are initiated.

Intracellular recordings taken from muscle cells beyond the regions of fast and slow conduction revealed hyperpolarizing potentials which were sometimes followed by low amplitude depolarizations. For the DE2 motoneuron, hyperpolarizing responses were observed in muscle cells anterior to the commissure (Fig $5 b$ ). Activating either DE1 or DE3 motoneurons produced similar regions of muscle hyperpolarization posterior to their zones of slow conduction and anterior to their zones of fast and slow conduction vclocitics (Fig. $5 a$ ).

Recordings taken from muscle cells posterior to the DE1 neuron, in the region of slow conduction, occasionally revealed a depolarizing potential which was followed immediately by a hyperpolarization. Injecting hyperpolarizing current into muscle cells in this region blocked the posterior propagation of the depolarizing response but left the hyperpolarization intact (Fig. 6). This result implies that the hyperpolarizing response is produced by activity in a neuron (presumably a DI motoneuron).

\section{Discussion}

\section{Conduction velocity measurements}

In nematode locomotion, waves of flexion in the ventrodorsal plane are propagated along the hody. In Ascaris lumbricoides, these waves typically propagate at about $1 \mathrm{~cm} / \mathrm{sec}$. Signals in the dorsal motoneurons which are generated in the ventral nerve cord will be carried to the dorsal nerve cord across the commissure and propagate anteriorly or posteriorly (depending on neuronal type). One simple model for neural control of nematode locomotion would suggest that the rate of wave propagation is equivalent to the rate of neuronal signal propagation. Earlier investigations on intact ventral neuromuscular preparations (de Bell et al., 1963; Weisblat and Russell, 1976) a

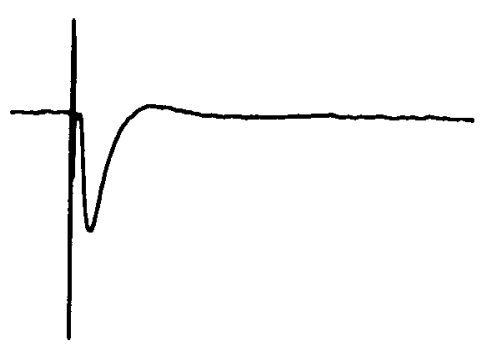

\section{b}

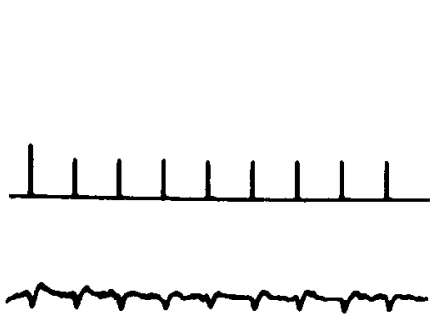

Figure 5. Stimulating excitatory motoneurons evokes hyperpolarizing responses in muscle cells they do not directly innervate. $a$, Response recorded in muscle cell posterior to the region of depolarizing responses evoked by stimulation of a DE1 motoneuron. The average of 64 responses is shown. $b$, Hyperpolarizing responses evoked by stimulation of a DE2 $2_{1}$ motoneuron recorded from dorsal muscle cells anterior to the DE2 commissure. Top trace, stimulus monitor; bottom trace, muscle potential recording.

indicated that conduction velocities were too fast to account for the rate of wave propagation; however, these studies did not determine the conduction velocities of individual neurons. In the experiments described in this paper, we measured the conduction velocities of activity evoked by single identified motoneurons. For all classes of dorsal motoneurons, signals propagating at about $28 \mathrm{~cm} / \mathrm{sec}$ are evoked in muscle cells directly innervated by the neuron. These conduction velocities are much too fast to fit the simple model of one signal-one wave.

In the case of the DE1 and DE3 neurons (and rarely for the DE2 neurons), there is also evoked muscle activity in regions not directly innervated by the stimulated neuron. These signals travel at a velocity of about $13 \mathrm{~cm} / \mathrm{sec}$. Our evidence suggests that these slowly propagating signals are carried by the muscle syncytium, whereas the rapidly propagating activity travels along the neuron. Thus, muscle cells can be activated either directly, via chemical synapses, or indirectly, via electrical 


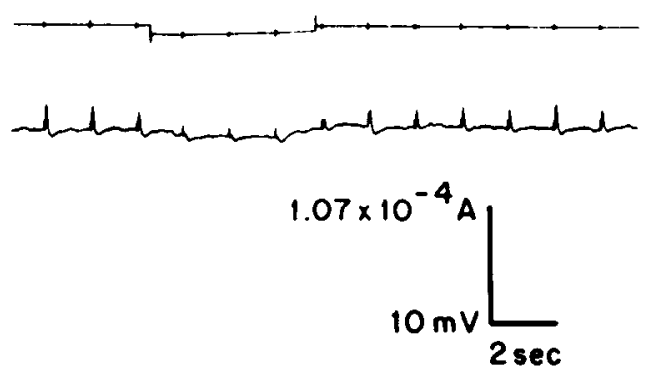

Figure 6. Current injection blocks the slowly propagating depolarization but not the hyperpolarization evoked by stimulation of a DE1 motoneuron. Placement of stimulating, recording, and current injection electrodes was as described in Figure 3. Injecting hyperpolarizing current into muscle cells posterior to the DE1 commissure blocked the slowly propagating depolarizing potentials but left the hyperpolarization intact. Depolarizations are conducted through the electrically coupled muscle cells, whereas hyperpolarizations result from neuronal activity. Top trace, current monitor; bottom trace, muscle response. The vertical scale shows the calibration for the top and bottom traces, respectively.

synapses which connect them to the directly activated muscle cells.

Recordings taken from the muscle cells located in the anatomically defined output region of the stimulated neuron revealed a transition from the fast conduction velocity to a slower velocity that was similar to that recorded for syncytial conduction. We believe that the slower velocity represents syncytial conduction and results from either a failure of the neuronal signal to invade the distal portions of the axon or a reduction in the frequency of neuromuscular synapses in and beyond the transition region. When there was a transition in the conduction velocity, we fitted the data to two straight lines (Figs. 3 and 4) although, because of scatter, we cannot exclude more complex interpretations. In particular, the transition from fast to slow conduction may not be abrupt. Indeed, one would expect a transition zone where there is interaction between the neuronal (fast) and electrical (slow) systems. These findings are consistent with the results of experiments in which the synaptic contacts made by each DE neuronal type onto VI dendrites are mapped physiologically (Walrond and Stretton, 1985). The region of fast conduction of a DE motoneuron corresponds to the region where it activales VI dendrites, but in the regions of slow conduction, VI dendrites are not activated.

Other investigators have studied the conduction velocities of electrical signaling in the Ascaris nerve cords and have found different numerical values, ranging from 6 to $22 \mathrm{~cm} / \mathrm{sec}$. Weisblat and Russell (1976) showed that anodal stimulation of the ventral nerve cord evoked hyperpolarizing responses which propagated at $22 \mathrm{~cm} / \mathrm{sec}$, whereas cathodal stimulation evoked hyperpolarizing responses propagating at $16 \mathrm{~cm} / \mathrm{sec}$. They found that removing muscle cell bodies along the conduction path sometimes blocked the fast conducting responses but left the slow conducting responses intact, and they concluded that the faster conduction was mediated by electrically coupled muscle cells, whereas the slower conduction was in neurons. In contrast, we have concluded that neuronal conduction is faster than muscle conduction.

Weisblat and Russell (1976) were unaware of synaptic interactions which confound the interpretations of their results, and we believe that neuronal conduction accounts for both the fast and slow conducting responses which they describe. Two features of their results have to be explained: the origin of the fast and slow conduction and the effects of plucking muscle cells on fast conduction. The putative ventral excitatory motoneurons V-1 and V-2 (see "Discussion" in Walrond et al., 1985) receive synaptic input from the large nonsegmental interneu-

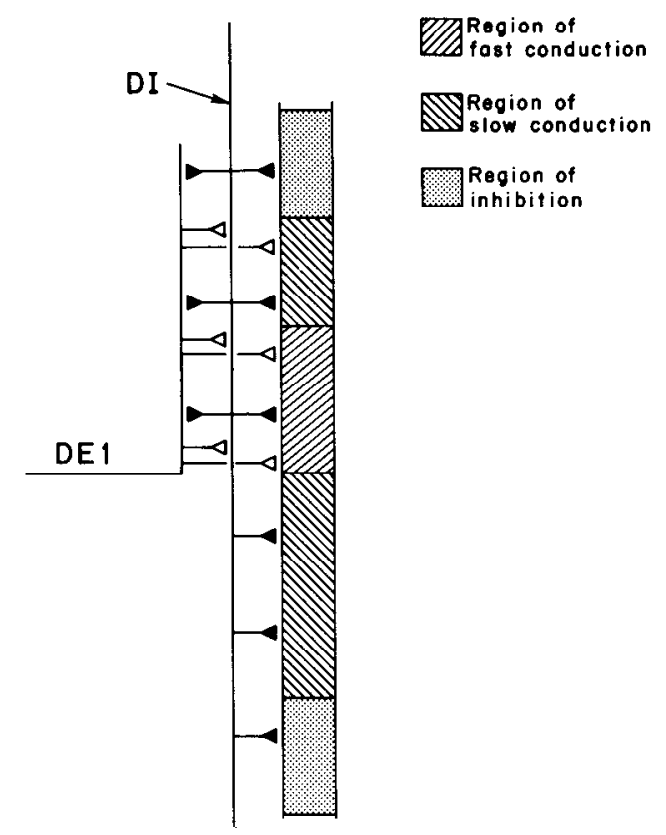

Figure 7. A diagram of the synaptic interactions between the DE1 motoneuron, the DI motoneuron, and muscle. The DE1 neuron has excitatory synaptic output onto muscles and the DI axon (open triangles). The DI neuron synapses onto muscle and the DE1 axon (solid triangles). Activation of the $\mathrm{DE} 1$ neuron produces anterior to the commissure a zone of fast conduction, a zone of slow conduction, and a zone of inhibition. Posterior to the commissure there is a zone of slow conduction and a flanking zone of inhibition.

rons. The fast conducting depolarizing response may represent activity, conducted through these large interneurons, which synaptically activates the excitatory motoneurons (Kass et al., 1980). On the other hand, the VI motoneurons, which are smaller in diameter, do not receive synaptic input from the interneurons. The slower conduction velocity observed for the hyperpolarizing potentials may therefore represent the conduction velocity of the VI motoneurons. Differences in the absolute values of conduction velocities in our experiments and theirs might be expected since we stretched the dissected preparation to its original length in the intact worm and they did not. The effect of plucking muscle cells on fast conduction may result from damage to the neurons, since plucking muscles near the nerve cords often blocks neuronally conducted activity (R. Davis, personal communication). Finally, since in Weisblat and Russell's (1976) preparation the ventral nerve cord was connected to the dorsal cord by commissures, a neural pathway bypassing the region where muscles were plucked remained intact. Ventral stimulation of a DE motoneuron may have activated VI motoneurons through synaptic connections in the dorsal cord (Walrond and Stretton, 1985). This connection would not have been disrupted by damage to ventral neurons or muscle located in the region plucked free of muscle.

\section{Dorsal inhibition mediated by $D E$ axons}

Ventrally activating DE motoncurons cvoked both depolarizing and hyperpolarizing responses in the dorsal musculature. We suspect that the inhibitory responses recorded in regions outside the zones of fast and slow conducting depolarization result from the synaptic activation of the DI motoneuron. Ultrastructural studies of the dorsal cord have revealed synapses from the axons of all three types of DE motoneurons onto the axons of the DI motoneurons (P. A. Desnoyers, J. E. Donmoyer, and A. O. W. Stretton, unpublished observation). If these synapses are excitatory, then this local circuit inter- 

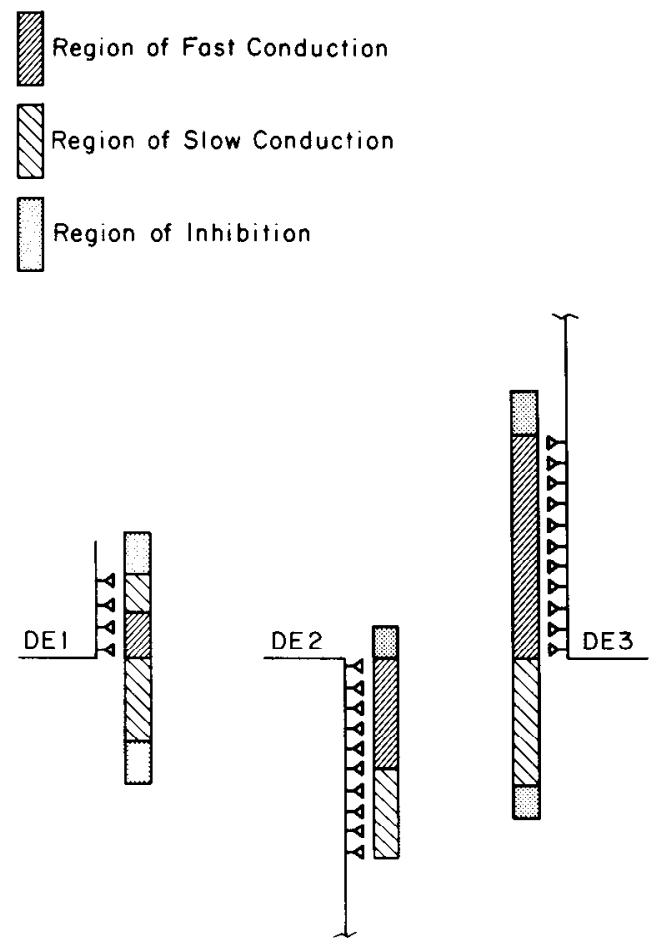

Figure 8. Summary of the regions of fast and slow conducting depolarization and of hyperpolarization produced by stimulation of DE1, DE2, and DE3 neurons. Within the neuromuscular output zones there are regions of both fast and slow conduction. In the case of the $\mathrm{DE} 1$ and $\mathrm{DE} 3$ neurons there are also regions of slow conduction in muscle cells not directly innervated by these neurons. The regions of fast conduction correspond to the neuromuscular output zones. The regions of slow conduction result from activity propagating through the electrically coupled muscle cells. The flanking regions of inhibition are probably produced by synaptic activation of the DI motoneuron (see Fig. 7) by the DE neuron.

action should activate the inhibitory axon whenever a dorsal excitor is turned on.

The relative position of the excitatory and inhibitory axons, together with the conduction velocities of neuron and muscle, may explain how activating a single excitor produced zones of excitation and inhibition. For example, if the DE1 neuron activated the DI axon along their coextensive region (Fig. 7), the synaptically evoked activity would propagate anteriorly and posteriorly along the inhibitory motoneuron. Due to synaptic delay, the activity conducted anteriorly along the DI neuron would follow the neuronally evoked excitatory activity. Since the output to muscle of the DI neuron extends beyond the neuromuscular output region of the DE neuron, inhibitory responses would be expected to occur outside the region of the fast conduction (Fig. 7). Once the activity propagating rapidly along the inhibitory motoneuron overtakes the slow excitatory activity propagating either anteriorly or posteriorly through the muscle syncytium, further propagation through the muscles would be inhibited. Beyond this point there would be a region of hyperpolarized muscle cells due to inhibitory input from the DI axons.

One interesting consequence of these flanking zones of inhibition found for DE1 and DE3 is that they block invasion of the entire muscle field by the excitatory responses from single neurons. Since gap junctions between muscle cells appear to provide a continuous electrical pathway along the entire length of the worm, a mechanism to block propagation of excitatory responses would be necessary for the production of a waveform in which dorsal and ventral muscle cells are contracted in different regions of the body at the same time.

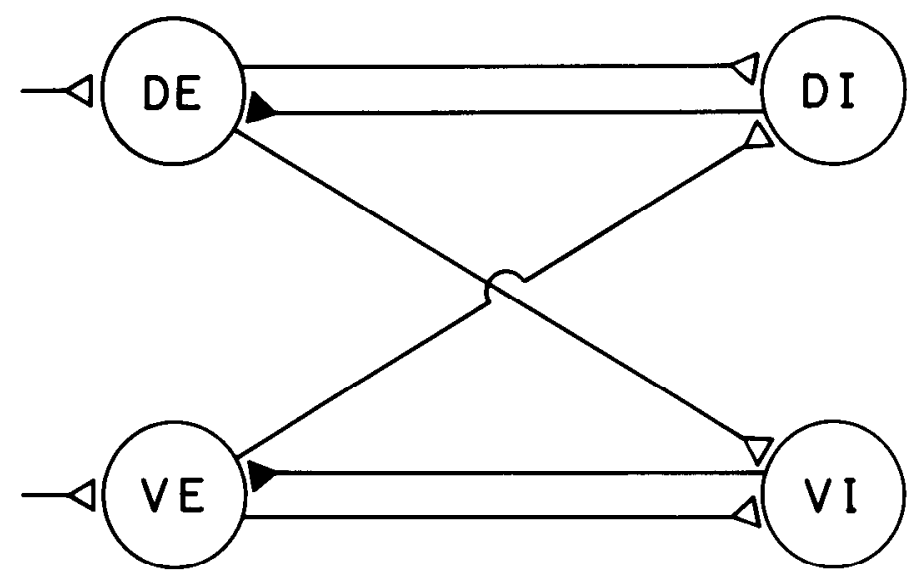

Figure 9. Model for a motoneuron oscillator. Reciprocal inhibition occurs between the DE and VI neuron and between the VE and DI neuron. Reciprocal synapses also occur between the DE and DI neurons and between the VE and VI neurons. Open triangles show excitatory synapses and solid triangles show inhibitory synapses. The excitatory input to DE and VE cells from interneurons is tonically active. The circuit consists of two feedback loops (DE $\rightleftarrows \mathrm{DI}$, and $\mathrm{VE} \rightleftarrows \mathrm{VI}$ ). Activity in the excitors persists until after a delay; inhibitury feedback turns them off. The dorsal and ventral excitors are kept out of phase by the reciprocal inhibitory connections between the two nerve cords $(\mathrm{DE} \rightarrow \mathrm{VI}, \mathrm{VE} \rightarrow \mathrm{DI})$. Physiological evidence exists for the excitatory nature of the DE $\rightarrow$ VI synapse (Walrond and Stretton, 1985) and for the DE $\rightarrow$ DI synapse (this paper). Other synapses are based upon electron microscopy. The presence of time delays in the feedback circuits (due, for example, to use-dependent properties of synapses or slow channels) is an important prediction of this model.

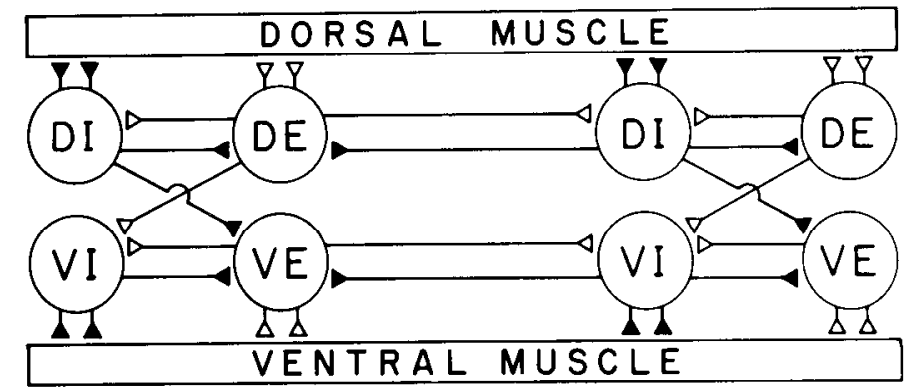

Figure 10. Two linked oscillators and a pathway connecting them. Two oscillatory units (Fig. 9) have been linked through same-side synaptic interactions (DE and DI dorsally and VE and VI ventrally). Such a linkage can be used to explain the propagation of the body bend. The elements required for this scheme have been identified by electron microscopy.

The behavior of the DE2 neuron is strikingly different from that of the DE1 and DE3 neurons. Upon activation of the DE2 neuron, we did not usually detect a region of slow conduction between the fast conducting zone and the anterior region of inhibition (Fig. 8) (in the single example shown in Table I, the region of slow conduction was short). Furthermore, there was no region of inhibition detectable posterior to the excitatory activity, and the inhibitory activity anterior to the commissure was much stronger than that observed for DE1 and DE3 neurons. We have no satisfactory explanation for these differences. Possibly they reflect differences in the total number and strength of the synaptic contacts between the DE and DI neurons (DI receives more synapses from DE2 than from DE1 or DE3).

\section{Coordination of the locomotory system}

Elementary postures produced by single motoneurons. As described in the preceding paper (Walrond and Stretton, 1985), 
dendrites of the VI motoneurons receive cxcitatory input from the axons of the DE neurons. The ventral output zone of the VI neuron and the dorsal neuromuscular output zone of the excitor that turns it on affect the same region of the worm. Therefore, stimulating a single DE neuron produces, at the same location along the length of the worm, a region of dorsal excitation and an opposing zone of ventral inhibition by this reciprocal inhibitory circuit (see Fig. 5 in Walrond and Stretton, 1985). In this paper we have shown that stimulating a single DE neuron produces yet further effects: the region of dorsal muscle excitation is flanked by regions of dorsal inhibition. Taken together with the system of reciprocal inhibition, the activation of each DE1 and DE3 should produce an omega $(\Omega)$ shape in the body of the worm; this would be formed by a zone of excitation flanked by two zones of inhibition in the dorsal musculature, and a complementary zone of ventral inhibition. These omegas could be the elementary postures of which the body waveform is composed. Each excitor could generate such an elementary posture at a different location along the animal. An alternating chain of dorsal and ventral omegas, properly phased, would throw the body into a smooth waveform (for example, the flanking inhibition of a ventral omega might coincide with and reinforce the reciprocal inhibition of the neighboring dorsal omega).

Propagation of the waveform. For propagation of the waveform to occur, the location of dorsal and ventral omega figures must change in space and time. Since each motoneuron is present in multiple copies, repeated serially along the length of the worm, sequential activation of members of a given class could lead to propagation of the omega figures. We now have to explain how the sequential activation occurs. It is tempting to suppose that the interneurons play a central role since they are presynaptic to the excitatory motoneurons. (The inhibitory motoneurons receive their input from excitatory motoneurons.) Three possible roles for the interneurons come to mind.

$a$. The interneurons may play an instructive role, in which individual interneurons encode preprogrammed postures consisting of alternating dorsal and ventral omega figures. If different interneurons coded for postures corresponding to different phases of the waveform, switching on these interneurons in the appropriate order could lead to propagating waves. Although this model cannot yet be ruled out, our electron microscopic results (J. E. Donmoyer, P. A. Desnoyers, and A. O. W. Stretton, unpublished observations) do not support the scheme. The necessary alternation of synaptic input from interneurons to dorsal and ventral excitors has not been observed.

$b$. The interneurons could play a permissive role, acting as gates that allow or disallow activity in sets of motoneurons. Once activated, interactions between the excitatory and inhibitory motoneurons may be sufficient to produce patterned output. One such possible scheme, which uses the synaptic contacts that have been observed by electron microscopy, is shown in Figure 9. This circuit can act as an oscillator which produces alternating dorsal and ventral excitatory activity. Anatomical results have shown that the excitatory and inhibitory neurons of adjacent uscillators overlap, so that neighboring oscillators are linked by shared elements (Fig. 10). Possibly these connections result in the coupling of neighboring oscillators with appropriate phase delays.

c. The interneurons may play no role in locomotory behavior (but perhaps are involved in other behaviors). In this case, the interactions among motoneurons would be the sole source of patterning and of the initiation of propagation.

To test the validity of these or more complex models will require much more detailed knowledge of the electrophysiological properties of the neurons and their synaptic connections.

\section{References}

Crofton, H. D. (1971) Form, function and behavior. In Plant Parasitic Nematodes, B. M. Zuckerman, W. F. Mai, and R. A. Rhode, eds., Vol. III, pp. 83-116, Academic Press, Inc., New York.

de Bell, J. T., J. del Castillo, and V. Sanchez (1963) Electrophysiology of somatic muscle cells of Ascaris lumbricoides. J. Cell Comp. Physiol. 62: $159-177$.

del Castillo, J., W. C. de Mello, and T. Morales (1967) Initiation of action potentials in the somatic musculature of Ascaris lumbricoides. J. Exp. Biol. 46: 263-279.

Kass, I. S., C. C. Wang, J. P. Walrond, and A. O. W. Stretton (1980) Avermectin $\mathrm{B}_{1 a}$, a paralyzing anthelmintic that affects interneurons and inhibitory motoneurons in Ascaris. Proc. Natl. Acad. Sci. U. S. A. $77: 6211-6215$.

Rosenbluth, J. (1965) Ultrastructure of somatic muscle cells in Ascaris lumbricoides. J. Cell Biol. 26: 579-591.

Stretton, A. O. W., R. M. Fishpool, E. Southgate, J. E. Donmoyer, J. P. Walrond, J. E. R. Moses, and I. S. Kass. (1978) Structure and physiological activity of the motoncurons of the nematode Ascaris. Proc. Natl. Acad. Sci. U. S. A. 75: 3493-3497.

Walrond, J. P., and A. O. W. Stretton (1985) Reciprocal inhibition in the molor nervous system of the nematode Ascaris: Direct control of ventral inhibitory motoneurons by dorsal excitatory motoneurons. J, Neurosci. 5: 9-15.

Walrond, J. P., I. S. Kass, A. O. W. Stretton, and J. E. Donmoyer (1985) Identification of excitatory and inhibitory motoneurons in the nematode Ascaris by electrophysiological techniques. J. Neurosci. 5: $1-8$.

Weisblat, D. A., and R. L. Russell (1976) Propagation of electrical activity in the nerve cord and muscle syncytium of the nematode Ascaris lumbricoides. J. Comp. Physiol. A. 107: 293-307. 